\title{
Hypothermic Machine Perfusion in Kidney Transplantation: Back to the Future?
}

\author{
Mariano Ferraresso ${ }^{1,2^{*}}$ and Evaldo Favi $^{2}$ \\ ${ }^{1}$ Division of Kidney Transplantation, Fondazione Cà Granda IRCCS, Ospedale Maggiore Policlinico, Milan, Italy \\ ${ }^{2}$ Department of Clinical Sciences and Community Health, University of Milan, Italy
}

*Corresponding author: Mariano Ferraresso, Division of Kidney Transplantation, University of Milan, Milan, Italy, Tel: +39 0250320393; Fax: +39 0250320384; E-mail: mariano.ferraresso@unimi.it

Received date: January 26, 2016; Accepted date: January 28, 2016; Published date: February 04, 2016

Copyright: ( $) 2016$ Ferraresso M, et al. This is an open-access article distributed under the terms of the Creative Commons Attribution License, which permits unrestricted use, distribution and reproduction in any medium, provided the original author and source are credited.

\begin{abstract}
Static cold storage is currently the most used method of organ preservation worldwide. However, cutting edge technology and dramatic changes in the donor pattern have lately renewed the interest toward hypothermic machine perfusion. Marginal and cardiac death donors show higher rates of primary non function and delayed graft function compared to standard criteria donors. In this setting, machine perfusion may offer several theoretical advantages such as improved organ preservation, continuous graft evaluation, and ex-vivo conditioning of the graft before implantation. These topics have been recently reassessed by several studies. In particular, perfusion characteristics (renal resistance) and perfusate biomarker concentrations (lactate dehydrogenase, aspartate aminotransferase, heart-type fatty acid binding protein, and IL-18) during machine preservation, proved to be reliable tools to rule out graft viability and predict outcomes after transplantation. Treatment strategies acting on tissue repair, cell metabolism, and allorecognition pathway are also under investigation with promising results. Machine perfusion has finally shown its real potential however, stronger evidences and updated cost-effectiveness analysis are needed to fully support its role for the next future.
\end{abstract}

Keywords Hypothermic machine perfusion; kidney transplantation; organ preservation; organ assessment

\section{Abbreviations \\ DBD: Donation after Brain Death; ECD: Expanded Criteria Donor; DCD: Donation after Cardiac Death; PNF: Primary Non Function; DGF: Delayed Graft Function; HMP: Hypothermic Machine Perfusion; GS: Graft Survival; RR: Renal Resistance; LDH: Lactate Dehydrogenase; AST: Aspartate amino-Transferase; GST: Glutathione- S-Transferase; HFABP: Heart-type Fatty Acid Binding Protein; NAG: N-Acetyl- $\beta$-D-Glucosaminidase; MDA: Malondialdehyde.}

\section{Introduction}

Kidney transplantation is the treatment of choice of end stage renal disease [1]. Impressive advances in organ procurement, surgical technique, post-operative care, and immunosuppression have significantly reduced transplant-related morbidity and mortality transforming an extraordinary event in a safe and routinely performed procedure.

In an attempt to expand the donor pool and match the increasing amount of patients in the waiting lists, the transplant community has more recently moved from the traditional boundaries set by the standard donation after brain death (DBD) toward the new frontiers of expanded criteria donors (ECD) [2,3] and donation after cardiac death (DCD) $[4,5]$. However, compared to kidneys retrieved from standard donors, ECD and DCD organs show higher rates of primary failure (PNF) and delayed graft function (DGF) [3-6]. Inferior renal function and higher discard rates have been also highlighted by several reports. In this setting, optimal preservation, reliable assessment, and possibly ex-vivo conditioning of the graft before implantation become extremely important.

Cold storage (literally in ice) has been serving our cause for almost sixty years. Ideal organ quality, favorable transport requirements, and economic reasons have been the main factors behind the supremacy of this preservation modality. Nevertheless, the dramatic changes observed in the transplantation scenario during the last two decades combined with modern technologies, have once again raised interest into an old but renewed ally: hypothermic machine perfusion (HMP).

First introduced into the clinical practice by F.O. Belzer in the late sixties, the new generation of portable hypothermic perfusion machines offers several advantages over traditional hypoxic flush preservation and cold storage $[7,8]$.

\section{Organ Preservation}

From the physiological point of view, controlled hypothermia $\left(4-10^{\circ} \mathrm{C}\right)$ allows homogeneous cooling of the kidney with reduced but still preserved metabolism. Such a low degree of cellular activity does actually help tissues continue the reparative process triggered by the retrieval and does improve the ability of the organ to effectively cope with the future damages of the ischemia-reperfusion injury. The opportunity to deliver oxygen to the cells through normal air tension, external oxygenation, or even oxygen carriers released into the preservation fluid, represents another important feature of machine perfusion. Oxygen supply is needed to keep mitochondrial function ensuring adequate energy production and correct ionic balance during storage. Moreover, continuous or pulsatile perfusion granted by the machine, prevents the collapse of the capillary bed with a wider distribution of the preservation solution and a more effective washout of toxic metabolites, clots, and debris.

All those theoretical benefits have been recently investigated in three randomized clinical trials in DBD, ECD, and DCD kidney transplantation. In their original work dated 2009 [9] and in the extension trial published in 2012 [10], Moers and colleagues observed significantly lower rates of DGF and better graft survival in DBD and ECD kidneys preserved with hypothermic machine perfusion compared to cold storage (3-year GS: $91 \%$ vs. $86 \%$ and $86 \%$ vs. $76 \%$, 
respectively). Beneficial effects of HMP were more evident in transplants from marginal donors and for DBD transplants not experiencing DGF. Lower rates of DGF were also reported for machine-perfused DCD kidneys, but the study failed to demonstrate any survival advantages in this subgroup of recipients. Conflicting results were reported by two other trials addressing the same topics in Maastricht Category III DCD donors. In the first trial [11], performed under Eurotransplant, 155 kidneys pairs were randomized to immediate machine perfusion or static cold storage. DGF was observed in $53.7 \%$ of the organs allocated to HMP and in $69.5 \%$ of the graft preserved in ice $(p<0.05)$. Functional DGF (i.e. absence of a decrease in serum creatinine level by a minimum of $10 \%$ per day during three consecutive days in the first post-operative week) showed a similar trend $(19.5 \%$ vs. $51.2 \%$, respectively). There were no differences in PNF, acute rejection, and 1-year graft survival rates between the two groups. Similar 1-year graft survival (93.3\% vs. 97.8\%) and renal function were reported by the randomized clinical trial performed in UK in 2010 [12]. Surprisingly, the study showed no differences in DGF rates between HMP and cold storage (58\% vs. 56\%) but the fact that some of the kidneys allocated to HMP were actually put on ice before effective pumping may somehow explain this unexpected finding.

Although more updated cost-effectiveness analysis are still needed [13], current trends seem to support the use of HMP for DBD kidneys [14], especially from marginal donors [15]. Indeed, kidneys preserved on HMP show lower rates of DGF compared to cold storage and it is well recognized that DBD and ECD transplants experiencing DGF do have an increased risk of acute rejection, poor graft function, and graft failure [16]. As a matter of fact, DCD kidneys have higher rates of PNF and DGF compared to age-matched DBD organs. However, for some reason still unknown, DGF does not significantly impact long term DCD transplant outcomes [17]. It has been postulated that organ damages caused by the events initiated by brain death can be very different from the ones potentially triggered by cardio-circulatory death $[18,19]$. As a consequence, HMP may not be actually able to address the specific needs of DCD kidneys during preservation. Theoretically, uncontrolled DCD (mainly Maastricht Category I and II) may exhibit a different behavior compared to controlled DCD (Category III) but good quality studies in this field have yet to be published.

\section{Organ Assessment}

HMP allows real time evaluation of the organ before implantation through tissue sampling, continuous assessment of the quality of the perfusion, and timely analysis of the perfusate. The possibility to test the kidney during machine preservation is not something new as studies addressing this issue were already available thirty years ago. Nevertheless, modern technologies combined with current understanding of cell biology can offer a complete different set of information compared to the past.

Several studies have suggested that perfusion parameters during machine preservation can predict transplant outcomes but until recently almost only retrospective analysis have been performed. In his prospective trial evaluating HMP parameters in DBD, ECD, and DCD kidney transplants [20] Jochmans demonstrated that vascular renal resistance (RR) is an independent risk factor for DGF and 1-year graft failure. However, no reliable cut off value truly predictive of DGF could be found and there was no correlation between RR and PNF. In other two prospective studies [21,22]. Yushkov showed that RR $>0.3$
$\mathrm{mmHg} / \mathrm{mL} / \mathrm{min}$ is associated with poor 1-year graft survival. Higher rates of PNF were described by de Vries and his group [23] in Maastricht Category III DCD kidneys with high RR early during machine perfusion.

Those results seem to validate RR for organ assessment but need to be cautiously interpreted as unable to provide a definitive parameter that could be reliably used to evaluate graft viability and function before implantation [24]. Avoiding unsuccessful transplants is obviously a major concern but the risk we may discard good quality kidneys remains too high if $\mathrm{RR}$, is not combined with other prognostic factors $[25,26]$. Indeed, more powered studies are still required to definitely address this issue.

Another strong point of HMP is the opportunity to further evaluate organ viability and quality through non-invasive analysis of the composition of the perfusate during preservation. Ideally, perfusate biomarkers can give us dynamic information on previous organ damage, active metabolism, ongoing reparative process, and expected transplant outcomes. At the moment, perfusate testing is mainly based on bio-chemical methods but more sophisticated assays such as proton-nuclear magnetic resonance spectroscopy are under investigation [27]. Many molecules have been studied so far but only few showed significant association with important post-transplant end points in multivariate analysis [28]. Lactate dehydrogenase (LDH) and aspartate aminotransferase (AST), well known non-specific markers of tissue damage, proved to be highly predictive of DGF in DBD and DCD kidney transplants [29]. Associations between PNF and graft function have been also reported but evidences remain overall weak. Glutathione-S-transferase (GST), a more specific marker of renal tubular injury, and Heart-type fatty acid binding protein (HFABP), generally released under ischemic conditions, have also shown good correlations with DGF [29]. Other biomarkers of potential interest as implicated in the process of ischemia/reperfusion injury are $\mathrm{N}$-acetyl$\beta$-D-glucosaminidase (NAG) [29], malondialdehyde (MDA) [30], redox-active iron [31], and IL-18. IL-18 in particular, released by mononuclear cells in response to oxidative stress, has been associated with PNF in controlled and uncontrolled DCD kidney transplants [28].

Despite promising results, perfusate biomarkers as well as perfusion parameters cannot be considered an absolute indicator of graft viability. Combining different biomarkers is the best strategy to overcome their individual lack of sensitivity and specificity but the risk of bursting money without having a definitive answer to our question remain significant. Too many variables do play a role before, during, and after preservation and probably the best way to assess a kidney is to consider donor characteristics, macroscopic evaluation, histology, HMP parameters, and perfusate biomarkers all together. A comprehensive score taking into account all these information can more likely find its place as a reliable albeit not definitive toll for helping clinicians discard not viable organs, chose the right kidney for the right recipient, and select the most appropriate immunosuppressive treatment after transplantation [32-35].

\section{Ex-vivo Conditioning}

Administration of different oxygen concentrations has been the first step toward more complex attempts to improve transplant outcomes through direct conditioning of the graft during preservation $[36,37]$. Experimental models addressing how to deliver cytoprotective mixtures, immune modulators or even immunosuppressive agents 
during HMP so as to prevent or reduce ischemia-reperfusion injury and rejection are under development [38-40]. In the future, stem cells treatment [41] and targeted gene therapy [42] toward donor organs will probably make the difference between the old age and the new era of transplantation but, at the moment, reports remain anecdotal.

\section{Conclusion}

HMP is the ideal methods of preservation for DBD and ECD kidneys as it shows lower rates of PNF and DGF compared to cold storage. Further studies are warranted to definitely address this issue in DCD kidney recipients. Renal resistance during preservation is significantly associated with important transplant outcomes such as DGF and early graft survival but no reliable cut off values have been yet identified. More specific studies addressing this issue in controlled and uncontrolled DCD kidneys are therefore needed to reach firm conclusions. Perfusate biomarker concentrations, especially LDH, AST, HFABP and IL-18 showed good correlation with graft viability, PNF or DGF but their lack of specificity and sensitivity strongly suggests to match results with other donor and graft characteristics. Ex-vivo conditioning of the graft before implantation through stem cells transfer or targeted gene therapy is now technically possible but many years will pass before it will find a proper place in clinical practice. As soon as more updated cost-effectiveness analysis of HMP will be available we will finally understand that sometimes what comes from the past can more easily bring as to the future.

\section{References}

1. Wolfe RA, Ashby VB, Milford EL, Ojo AO, Ettenger RE, et al. (1999) Comparison of mortality in all patients on dialysis, patients on dialysis awaiting transplantation, and recipients of a first cadaveric transplant. $\mathrm{N}$ Engl J Med 341: 1725-1730.

2. Metzger RA, Delmonico FL, Feng S, Friedrich K. Port, James J. Wynn, et al. (2003) Expanded criteria donors for kidney transplantation. Am J Transplant 3 Suppl 4: 114-125.

3. Querard AH, Foucher Y, Combescure C, Dantan E, Larmet D, et al. (2016) Comparison of survival outcomes between Expanded Criteria Donor and Standard Criteria Donor kidney transplant recipients: a systematic review and meta-analysis. Transpl Int.

4. Cho YW, Terasaki PI, Cecka JM, Gjertson DW (1998) Transplantation of kidneys from donor whose hearts have stopped beating. N Engl J Med 338: 221-225.

5. Weber M, Dindo D, Demartines N, Ambühl PM, Clavien PA. et al. (2002) Kidney transplantation from donors without a heartbeat. N Engl J Med 347: 248-255.

6. Gok MA, Asher JF, Shenton BK, Rix D, Soomro NA, et al. (2004) Graft function after kidney transplantation from non-heartbeating donors according to Maastricht category. J Urol 172: 2331-2334.

7. Jochmans I, O'Callaghan JM, Pirenne J, Ploeg RJ (2015) Hypothermic machine perfusion of kidney retrieved from standard and high risk donors. Transpl Int 28: 665-676.

8. Fuller BJ, Lee CY (2007) Hypothermic perfusion preservation: the future of organ preservation revisited? Cryobiology 54: 129-145.

9. Moers C, Smits JM, Maathuis MH, Treckmann J, van Gelder F, et al. (2009) Machine perfusion or cold storage in deceased-donor kidney transplantation. N Engl J Med 360: 7-19.

10. Moers C, Pirenne J, Paul A, Ploeg RJ (2012) Machine Preservation Trial Study Group Machine perfusion or cold storage in deceased-donor kidney transplantation. N Engl J Med 366: 770-771.

11. Jochmans I, Moers C, Smits JM, Leuvenink HG, Treckmann J, et al. (2010) Machine perfusion versus cold storage for the preservation of kidneys donated after cardiac death: a multicenter, randomized, controlled trial. Ann Surg 252: 756-764.
12. Watson CJ, Wells AC, Roberts RJ, Akoh JA, Friend PJ, et al. (2010) Cold machine perfusion versus static cold storage of kidneys donated after cardiac death: a UK multicenter randomized controlled trial. Am J Transplant 10: 1991-1999.

13. Wight J, Chilcott J, Holmes M, Brewer N (2003) The clinical and costeffectiveness of pulsatile machine perfusion versus cold storage of kidneys from heart-beating and non-heart-beating donors. Health Technol Assess 7: 1-94.

14. O’Callaghan JM, Morgan RD, Knight SR, Morris PJ (2013) Systematic review and meta-analysis of hypothermic machine perfusion versus static cold storage of kidney allografts on transplant outcomes. Br J Surg 100: 991-1001.

15. Baoping Jiao, Shurong Liu, Hao Liu, Donghua Cheng, Ying Cheng, et al. (2013) Hypothermic machine perfusion reduces delayed graft function and improves 1-year graft survival of kidneys from expanded criteria donor: a meta-analysis. PLoS One 8: e81826.

16. Perico N, Cattaneo D, Sayegh MH, Remuzzi G (2004) Delayed graft function in kidney transplantation. Lancet 364: 1814-1827.

17. Singh RP, Farney AC, Rogers J, Zuckerman J, Reeves-Daniel A, et al. (2011) Kidney transplantation from donation after cardiac death donors: lack of impact of delayed graft function on post-transplant outcomes. Clin Transplant 25: 255-264.

18. Westendorp WH, Leuvenink HG, Ploeg RJ (2011) Brain death induced renal injury. Curr Opin Organ Transplant 16: 151-156.

19. Akhtar MZ, Huang H, Kaisar M, et al. (2015) Using an integrated -omics approach to identify key cellular processes that are disturbed in the kidney following brain death. Am J Transplant: Epub ahead of print.

20. Jochmans I, Moers C, Smits JM, Leuvenink HG, Treckmann J, et al. (2011) The prognostic value of renal resistance during hypothermic machine perfusion of deceased donor kidneys. Am J Transplant 11: 2214-2220.

21. Yushkov Y Y, Alvarez-Casas J, Dikman S, Ying A, Tajik W, et al. (2010) Machine measured renal resistance (MMRR) is the most sensitive tool for prediction of early renal allograft survival. Transplantation 90: 290.

22. Yushkov YY, Stern J, Ying A, Icitovic N, Dikman SH, et al. (2012) Identifying risk factors in renal allografts before transplant: machinemeasured renal resistance and posttransplant allograft survival. Prog Transplant 22: 175-182.

23. De Vries EE, Hoogland ER, Winkens B, Snoeijs MG, van Heurn LW, et al. (2011) Renovascular resistance of machine-perfused DCD kidneys is associated with primary non function. Am J Transplant 11: 2685-2691.

24. Guarrera JV, Goldstein MJ, Samstein B, Henry S, Reverte C, et al. (2010). 'When good kidneys pump badly': Outcomes of deceased donor renal allografts with poor pulsatile perfusion characteristics. Transpl Int 23: 444-446.

25. Sonnenday CJ, Cooper M, Kraus E, Gage F, Handley C, et al. (2003) The hazards of basing acceptance of cadaveric renal allografts on pulsatile perfusion parameters alone. Transplantation 75: 2029-2033.

26. Callaghan CJ, Harper SJ, Saeb-Parsy K, Hudson A, Gibbs P, et al. (2014) The discard of deceased donor kidneys in the UK. Clin Transplant 28: 345-353.

27. Guy AJ, Nath J, Cobbold M, Ludwig C, Tennant DA, et al. (2015) Metabolomic analysis of perfusate during hypothermic machine perfusion of human cadaveric kidneys. Transplantation 99: 754-759.

28. Hoogland ER, de Vries EE, Christiaans MH, Winkens B, Snoeijs MG, et al. (2013) The value of machine perfusion biomarker concentration in DCD kidney transplantations. Transplantation 95: 603-610.

29. Moers C, Varnav OC, van Heurn E, Jochmans I, Kirste GR, et al. (2010) The value of machine perfusion biomarkers for predicting kidney transplant outcome. Transplantation 90: 966-973.

30. Kosieradzki M, Kuczynska J, Piwowarska J, Wegrowicz-Rebandel I, Kwiatkowski A, et al. (2003) Prognostic significance of free radicals: mediated injury occurring in the kidney donor. Transplantation 75: 1221-1227.

31. De Vries B, Snoeijs MG, von Bonsdorff L, Ernest van Heurn LW, Parkkinen $\mathrm{J}$, et al. (2006) Redox-active iron released during machine perfusion 
Citation: Ferraresso M, Favi E (2016) Hypothermic Machine Perfusion in Kidney Transplantation: Back to the Future? J Transplant Technol Res 6: e135. doi:10.4172/2161-0991.1000e135

Page 4 of 4

predicts viability of ischemically injured deceased donor kidneys. Am J Transplant 6: 2686-2693.

32. Anglicheau D, Loupy A, Lefaucheur C, Pessione F, Létourneau I, et al. (2008) A simple clinic-histopathological composite scoring system is highly predictive of graft outcomes in marginal donors. Am J Transplant 8 : 2325-2334.

33. Rao PS, Schaubel DE, Guidinger MK, Andreoni KA, Wolfe RA, et al. (2009) A comprehensive risk quantification score for deceased donor kidneys: The kidney donor risk index. Transplantation 88: 231-236.

34. Kasiske BL, Israni AK, Snyder JJ, Skeans MA, Peng Y, et al. (2010) A simple tool to predict outcomes after kidney transplant. Am J Kidney Dis 56: 947-960.

35. Kosieradzki M, Danielewicz R, Kwiatkowski A, Polak WP, Wszola M, et al (2002) Early function of kidneys stored by continuous hypothermic pulsatile perfusion can be predicted using a new "viability index" Transplant Proc 34: 541-543.

36. Hendry W, Struthers N, Duguid W, Hopkinson WI (1968) Observations on kidney stored by continuous hypothermic perfusion and hyperbaric oxygen. Br J Surg 55: 431-436

37. Pegg D, Green C, Foreman J (1974) Renal preservation by hypothermic perfusion. II. The influence of oxygenator design and oxygen tension. Cryobiology 11: 238-247.
38. Gage FA, Vodovotz Y (2003) Normalization of nitric oxide flux improves physiological parameters of porcine kidneys maintained on pulsatile perfusion. Nitric Oxide 9: 141-147.

39. Okada K, Yamashita C, Okada M, Okada M (1996) Efficacy of oxygenated University of Wisconsin solution containing endothelin-A receptor antagonist in twenty-four-hour heart preservation. J Heart Lung Transplant 15: $475-484$.

40. Yang B, Hosgood SA, Bagul A, Waller HL, Nicholson ML, et al. (2011) Erythropoietin regulates apoptosis, inflammation and tissue remodeling via caspase- 3 and IL- $1 \beta$ in isolated hemoperfused kidneys. Eur J Pharmacol 660: $420-430$.

41. Van Raemdonck D, Neyrinck A, Rega F, Devos T, Pirenne J, et al. (2013) Machine perfusion in organ transplantation: a toll for ex-vivo graft conditioning with mesenchymal stem cells? Curr Opin Organ Transplant 18: 24-33.

42. Kim N, Woo DC, Joo SJ, Song Y, Lee JJ, et al. (2015) Reduction in Renal Ischemia-Reperfusion Injury in Mice by a Phosphoinositide 3-Kinase p110gamma-Specific Inhibitor. Transplantation 99: 2070-2076. 